\title{
FINITE PROPAGATION SPEED AND KERNELS OF STRICTLY ELLIPTIC OPERATORS
}

\author{
DAVID GURARIE \\ Department of Mathematics \\ Case Western Reserve Lniversity \\ Cleveland, Ohı 44106
}

(Received September 20, 1984)

ADSTRACT. We establish estinates of the resolvent and other related kernels and discuss $L^{2}$-theory for a class of strictly eiliptic operators on $\mathbf{R}^{n}$. The class of operators considered in the paper is of the form $A_{0}+B$ with the leading elliptic part $A_{0}$ and a "singular" perturbation $B$, whose coeficients have $L^{0}-t y p e$ and are modeled after schrödinger operators.

KEY WORDS AND PHRASES. İiiptic operator, resolven=, semigrouf, penturifzion sepies, Finite propagation speed. 1230 MATHEMATICS SUBJECT CLASSIFICATION CODES. 47C05; 3ESO5

1. INTRODUCIION.

In this paper we shali study the resolvent kerrel $R_{\zeta}(x, y)=(r-A)^{-1}$ and other related "iunctions of $A^{\prime \prime}$ fcr a class of strictly elliptic operaturs $A_{0}=\sum a_{\alpha}(r) D^{2}$ on $\mathbb{R}^{n}$ and their perzurbations $A_{0}+B$.

We are farticularly interested in $L^{\text {p }}$-theory of eliiptic operators, $1 \leqslant p \leqslant 0$, and typicai probiems cha: arise here are spectral properties of $A$, clcseciness, essential selíajcintass, accretivity, semigroup generation, etc. Underlying all those is the quisticn of cxistence and estimates of the resoivent kernel $R_{5}$.

Typically the estimate has the form of a convolution-type bound

$$
i \hbar_{r}(x, y) ! \leqslant c(\zeta) H\left(\vdots: \because-y_{i}\right)
$$

with an $\mathrm{L}^{\mathrm{i}}$-radial decreasirg functicn $\mathrm{H}$.

In the uniformily elliptic case kerzels of the cesoivent $k_{5}=(5-A)^{-i}$ and semigroup $K_{t}=e^{-t A}$ were studied extensively ([1], [2], [3] et a1.). In particular, Eidelman [1] derived the following radiai bound of $K_{t}$ 


$$
\left|k_{t}(x, y)\right| \leqslant c t^{-n / m} \exp \left(-\frac{|x-y|^{m^{\prime}}}{m_{\sqrt{t}}}\right)
$$

where $m=\operatorname{order} A, m^{\prime}=\frac{m}{m-1}$, and I. Gelfand and G. Shilov [2] applied this estimate to study generalized eigenfunction expansions of $A$.

In [4], [5] we obtained similar estimates in a different way starting with the resolvent kernel. The latter approach allows us to treat perturbations of $A$, and consequently operators with "nonregular" coefficients. We applied these results to $\mathrm{L}^{\mathrm{p}}$-spectral theory of elliptic operators.

In the present paper we extend the results of [4] to a class of strictly elliptic operators with possibly unbounded coefficients, obtained by linear deformations of uniformly elliptic symbols in the $\xi$-variable, $a=a(x, \xi) \rightarrow \tilde{a}\left(x, \delta_{x}(\xi)\right)$. Here $x \rightarrow \delta_{x}$ means a matrix function $\left(\delta_{i j}(x)\right)$ depending smoothly on $\mathrm{x} \in \mathbb{R}^{\mathrm{n}}$.

Natural examples of such deformations are second order elliptic operators $A=\sum a_{i j}(x) D_{i j}^{2}$. Indeed any quadratic form $a(x, \xi)=\sum a_{i j}(x) \xi_{i} \xi_{j}$ can be viewed as a deformation of the simplest one: $|\xi|^{2}+\left|\delta_{x}(\xi)\right|^{2}$ with $\delta_{x}=\left(a_{i j}(x)\right)^{\frac{1}{2}}$. Other examples appear as right (left) invariant operators on nilpotent Lie groups (see [6]).

For the sake of presentation we shall restrict ourselves to the simplest case of deformations when $\delta_{x}=\delta(x) I$ is scalar ("conformal dilations"). Such transformations correspond to a multiplication of the leading symbol of $A$ with a positive function $\delta(x)^{\mathrm{m}}, \mathrm{m}=$ order of $\mathrm{A}$. The dilating factor $\delta(\mathrm{x})$ is subject to a certain constraint, called "finite propagation speed" condition. Its precise definition is given in $\S 2$. This condition limits the rate of growth of $\delta$ at $\infty$, which can not exceed $0(|x|)$. In other words leading coefficients of $A$ can not grow faster than $|x|^{m}$ for $m-t h$ order operators.

Let us observe that growth restrictions on the leading coefficients are well known in both ordinary (Sturn-Liouville) theory, $A=-a(x) \frac{d^{2}}{d x^{2}}+\ldots$ (see for instance [7], Ch. 9) and also for partial differential operators $A=\sum a_{i j}(x) D_{i j}^{2}+\ldots$ ([8]). A sufficient condition for A to be well defined (essentially selfadjoint or Weyl's "limit circle case") is the divergence of the integral

$$
\int^{+\infty} \frac{\mathrm{dx}}{\sqrt{\mathrm{a}(\mathrm{x})}} \text { on } \mathbb{R} ; \int^{+\infty} \frac{\mathrm{dr}}{\sqrt{\mathrm{a}(\mathrm{r})}} ;\left(\mathrm{a}(\mathrm{r})=\sup _{|\mathrm{x}| \leqslant \mathrm{r}}\left\|\mathrm{a}_{i j}(\mathrm{x})\right\|\right) \text { on } \mathbb{R}^{\mathrm{n}} \text {. }
$$

The latter condition was called in [8] "finite propagation speed." Physically, $\|\mathrm{a}(\mathrm{x})\|$ can be interpreted as a "local propagation speed," then integrals (1.2) measure the "amount of time required to get from a finite point to $\infty$ ".

It is not clear to what extent(1.2)is necessary to get a well defined operator A. Results of [9], [10], [11] indicate that it might be "too restrictive," yet in special cases ([12], [13]) it proved to be necessary in a certain sense. 
The form of "finite propagation speed" used in our paper is close but somewhat stronger than (1.2). It is expressed in terms of derivatives of $\hat{j}(x)$ rather than integrals. Also the way we apply it in our setting differs from the standard method of "hyperbolic wave propagators" (cf. [8], [14]).

Under assumptions of $\S 2$ we show that $\mathrm{m}^{- \text {th }}$ order elliptic operators are well defined in different $L^{p}$-spaces $(1<p \leqslant \infty)$ and their kernels (resolvent, semigroup, etc.) share many properties of "constant coefficient" and "uniformly elliptic" kernels (cf. [15], [16], [5], [4]). There is one notabie exception however: the radial bound (1) central for the argument of [5], [4] is no longer valid. Instead we utilize another Fourier analytic tool - The Hardy-Littlewood maximal function:

$$
f \rightarrow f^{*}(x)=\sup _{r>0} \frac{C_{n}}{r^{n}} \int_{|y| \leqslant r}|f(x+y)| d y .
$$

Maximal functions appear in this context when "deformations" of symbols (of resolvent and related operators) are translated into "deformations" of kernels. As a result one gets kernels of the type

$$
\mathrm{K}_{\delta}(\mathrm{x}, \mathrm{y})=\hat{o}^{-\mathrm{n}}(\mathrm{x}) \mathrm{K}\left(\frac{\mathrm{x}-\mathrm{y}}{\delta(\mathrm{x})}\right) \text {; }
$$

where $K$ is $\mathrm{L}^{1}$-radial, $\delta(\mathrm{x})>0$ - the dilating factor. If $S(x) \leqslant c$ is bounded (uniformly elliptic case) $K_{\delta}$ is estimated by the usual "L'-dilation"

$$
K_{\delta}(x, y) \leqslant \frac{1}{c^{n}} K\left(\frac{x-y}{c}\right) \text {. }
$$

In our case (unbounded $S(x)$ ) $K_{\hat{o}}$ has no longer a single convoluticn type bound (1.4). But the maximal function (1.3) applies to give

$$
\left(K_{\delta} f\right)(x) \leqslant\|K\|_{1} f^{*}(x)
$$

The maximal operator $\mathrm{E} \rightarrow \mathrm{f}^{*}$ is well known to be $\mathrm{L}^{\mathrm{P}}(1<\mathrm{p}<\infty)$ (see for inst. [17]), which yields a bound for the $L^{p}$-operator norm of $k_{\delta}$. Such bounds are used throughout the paper as a substitute for the radial bound (1.1).

otherwise our argument is similar to [4].

First we construct the "free" resolvent $R^{0}=\left(\zeta-A_{0}\right)^{-1}, A_{0}$ is the ieading (homogenfous) part of A, via the "parametrix series"

$$
R_{\zeta}^{0}=K_{\zeta} \sum_{0}^{\infty} L_{\zeta}^{k}=K_{\zeta}\left(I-L_{\zeta}\right)^{-1} \text {. }
$$

Here $K$ is a pseudodifferential operator ( $\psi D D$ ) with symbol $\frac{1}{\zeta-a(x, \xi)}, a(x, \xi)$ being the leading symbol of $A$, and $L$ a $\psi D O$ of order $\leqslant-1$, whose symbol is computed explicitly $(\S 4)$. series

The "perturbed" resolvent $R=(\zeta-A)^{-1}$ is constructed via the perturbation

$$
R_{\zeta}=\sum_{0}^{\infty} R_{\zeta}^{0}\left(B R_{\zeta}^{0}\right)^{k}
$$


The coefficients of perturbation $B=\sum b_{\alpha}(x) D^{\alpha}$ are taken in $L^{p}$-classes. Such classes are well known in the theory of Schrödinger operators $-\Delta+V(x)$ (cf. [18]) and were studied for higher order operators in [15], [16], [5], [4]. In our case $b_{\alpha}(x)$ can be allowed certain growth at $\{\infty\}$ depending on $\delta(x)$. Such conditions are naturally formulated in terms of weighted $L^{p}$-spaces, $L_{w}^{p}$, with weights $w$, that are fractional powers of $\delta$. Also the $L^{p}$-domain of $A_{0}$ and $A$ cal be characterized as a weighted $L^{P}$-Sobolev space (Theorem 2 ).

The central result of the paper, Lemma 1, analogous to Lemma 1 of [5], gives conditions (in terms of $L^{p}$-classes of coefficients) for relative boundedness of $B$ with respect to $A_{0}$ and estimates the norm of $\mathrm{BK}_{\zeta}$. This result applies in $\S 4$ to sum series(1.5) and (1.6), and to derive different corollaries. Among them we get

a) ${ }_{L}^{\mathrm{P}}$-closedness of $\mathrm{A}=\mathrm{A}_{0}+\mathrm{B}$ and bounds on its $\mathrm{L}^{\mathrm{P}}$-spectrum;

b) a priori estimates and essential selfadjointness of $A$ in $L^{2}$;

c) resolvent summability, i.e., convergence $\zeta R_{\zeta} f(x) \rightarrow f(x)$ in $L^{p}$ and a.e.

d) existence of a strongly continuous holomorphic semigroup $\left\{e^{-t A}\right\} \operatorname{Re} t>0^{\circ}$

Let us remark that due to the maximal function techniques adopted in the paper the $L^{1}$-space is excluded from consideration. The $\mathrm{L}^{1}$-theory of integral kernels $\mathrm{K}_{\hat{\delta}}(\mathrm{x}, \mathrm{y})=\delta(\mathrm{x})^{-\mathrm{n}_{\mathrm{K}}} \mathrm{K}\left(\delta(\mathrm{x})^{-1}(\mathrm{x}-\mathrm{y})\right)$ with $\mathrm{K} \in \mathrm{L}^{1}$ poses an interesting harmonic analysis question. At the end of $\S 4$ we give two examples which have bearing on $\mathrm{L}^{1}$-theory.

In conclusion let us mention that the basic $L^{2}$-theory of elliptic operators ([19], [20], [3]) was extended later in a fairly general setting of pseudodifferential calculus ([21], [9], [22], [6]). But much less is known about $L^{p}, p \neq 2$. Two recent works that discuss specifically $L^{p}$-theory are those by Nagel and Stein [6] and Beals [23]. Both methods allow to treat a variety of "nonelliptic problems" (subelliptic Laplacians, degenerate elliptic operators, etc.), but remain entirely within the pseudodifferential frainework in the sense that symbols (cnefficients) are assumed sufficiently smooth.

Another trend ([15], [16], [5]) was to explore what "amount of nonregularity" of coefficients yields "well defined" ${ }_{L}{ }^{p}$-operators. The present paper continues the latter trend, but our approach seems to be extendable to various "nonelliptic" situations as well. This extension will be discussed elsewhere.

2. THE CLASS OF OPERATORS.

Throughout this paper we shall consider operators of the form $A=A_{0}+B$ with the leading elliptic part $A_{0}=\sum_{j} a_{\alpha}(x) D^{\alpha}$ and a "singular" perturbation $B=\sum_{|\alpha| \leqslant m} b_{\alpha}(x) D^{\alpha}$. $|\alpha|=m$

The following assumptions will be made on $A_{0}$ and $B$.

I. The leading part $A_{0}$ is assumed to be homogeneous of degree $m$ and have a reai symbol $a(x, \xi)$ obtained by the "deformation" of a uniformly elliptic symbol $\tilde{a}(x, \xi)$

$$
a(x, \xi)=\tilde{a}(x, \delta(x) \xi)=\delta^{m}(x) \tilde{a}(x, \xi) .
$$


Uniform ellipticity as usual means that

$$
\mathrm{C}_{1}|\xi|^{\mathrm{m}} \leqslant \tilde{a}(\mathrm{x}, \xi) \leqslant \mathrm{C}_{2}|\xi|^{\mathrm{m}}, \text { all } \bar{\xi} \text {. }
$$

The dilating factor $\delta(x)$ and the coefficients of $\tilde{a}(x, \xi)=\sum \tilde{a}_{\alpha}(x) \xi_{,}^{i}$ are subject to the following constraints

$$
\begin{aligned}
& \left|\delta^{(\beta)}(x)\right| \leqslant \text { const } \delta^{1-|\beta|} ;|\beta| \leqslant \mathrm{m} \\
& \left|\tilde{a}_{\alpha}^{(\beta)}(\mathrm{x})\right| \leqslant \text { const } \delta^{-|3|} ;|\beta| \leqslant \mathrm{m}
\end{aligned}
$$

Here and elsewhere $u^{(\beta)}$ denote a partial derivative $\mathrm{D}^{\beta^{\prime}} \mathrm{u}$. Condition (2.1) is close but somewhat stronger than the "finite propagation speed" condition [8]. The latter adjusted for $\mathrm{m}^{- \text {th }}$ order operators of the above type requires

$$
\int^{+\infty} \frac{\mathrm{dr}}{\delta(r)}=+\infty, \quad \delta(r)=\sup _{|x| \leqslant r} \delta(x)
$$

whereas (2.1) implies

$$
\left|\frac{\delta^{\prime}}{\delta}\right| \leqslant \frac{c}{\delta} \Rightarrow \int^{+\infty} \frac{d r}{\delta(r)} \geqslant\left.\ln \delta\right|^{+\infty}=+\infty .
$$

Both conditions do not allow $\delta(x)$ to grow faster than $0(|x|)$ as $x \rightarrow \infty$.

II. Perturbation $B=\sum \quad b_{\alpha}(x) D^{\alpha}$ has coefficients with local $L^{p}$-type $|\alpha| \leqslant m$

singularities, $b_{\alpha} \in \mathrm{L}_{10 c}^{\mathrm{P}_{\alpha}}$. For each term $b_{\alpha}(x) D^{\alpha}$ of $B$ we introduce its "fractional order"

$$
\mathrm{d}_{\alpha}=\frac{\mathrm{n}}{\mathrm{p}_{\alpha}}+|\alpha| \text { and require } \mathrm{d}_{\alpha} \leqslant \mathrm{m} .
$$

The latter is needed in order to have $B$ bounded relative to $A_{0}$.

We also need some control of $b_{\alpha}$ at $\{\infty j$, depending on $\delta$. A convenient way to express it is in terms of weighted $L^{P}$-spaces, $L_{w}^{P}=\left\{f: \int|f w|^{P} d x<\infty\right\}$. Precisely, we take $b_{\alpha} \in L_{w}^{P_{\alpha}}$ with the weight

$$
w=w_{\alpha}=\delta^{-d} \text {. }
$$

Let us notice that the above class of operators is closed under the adjunction $A \rightarrow A^{*}$, provided the coefficients of $B$ are sufficiently smooth, $\left(\left.b_{\alpha} \in \mathbb{C}^{p}\right|_{|\alpha|, w}\right.$ - the $L^{p}$-weighted Sobolev space of order $\left.|\alpha|\right)$. Indeed the adjoint of $a_{\alpha}(x) D^{\alpha}$ is

$$
\mathrm{D}^{\alpha}\left(\mathrm{a}_{\alpha} \cdot\right)=\sum_{0 \leqslant 3 \leqslant \alpha}\left(\begin{array}{l}
\alpha \\
\beta
\end{array}\right) a_{\alpha}^{(\alpha-\beta)} \mathrm{D}^{\beta} .
$$

Remembering that $a_{\alpha}=\delta^{m \tilde{a}_{\alpha}}$ with $\tilde{a}_{\alpha}$ and $\delta$ subject to (2.1) - (2.2), we estimate the $\beta$-order coefficient of the adjoint

$$
a_{\alpha}^{(\alpha-\beta)}=\sum_{\gamma^{\prime}+\gamma=\alpha-\beta}\left(\hat{o}^{m}\right){ }^{(\gamma)}\left(\tilde{a}_{\alpha}\right)\left(\gamma^{\prime}\right)
$$


as

$$
\left|a_{\alpha}^{(\alpha-\beta)}\right| \leqslant \delta^{m-|\gamma|} \delta^{-\left|\gamma^{\prime}\right|}=\delta^{m-|\alpha-\beta|}=\delta^{|\beta|}, \text { q.e.d. }
$$

So the lower order terms of $A^{*}$ coming from the top order coefficients: $b_{\alpha}=a_{\alpha}^{(\alpha-\beta)}$, are $L_{w}^{\infty},\left(w=\delta^{-|\beta|}\right)$, i.e. belong to the above class of perturbations. Similar argument applies to derivatives of the coefficients $b_{\alpha}$ of B. Namely, interpolating in 3 parameters: $P$ (for $L^{P}$-class of $b_{\alpha}$ ), Sobolev (fractional) order $s$ and the exponent $t$ of the weight $w=\delta^{t}$, one can show that

$$
b \in L_{s ; \delta}^{p} t \Rightarrow b^{(\gamma)} \in L_{s}^{q}-|\gamma| ; \delta^{t-|\gamma|} \text {, where } \frac{n}{q}=\frac{n}{p}+|\gamma|
$$

This implies in particular that the class of perturbations $A_{0}+B$ includes all symmetric operators with the leading part $A_{0}$. The latter is important both for the argument below and in various applications.

3. THE MAIN LEMMA.

In this section we shall prove the main Lemma, which gives condition for relative boundedness of the operator $B=b(x) D^{\alpha}$ with respect to $A_{0}$ of $\S 1$, and provides an estimate of the operator norm of the product $B \times$ "parametrix of $\left(5_{2}-A_{0}\right)^{-1, "}$ This result will enable us to prove the convergence of series (1.5) and (1.6) and to derive all consequences in $\S 4$.

Let $A_{0}=a(x, D)$ be an elliptic operator of $\S 2$ with symbol $\mathrm{a}(\mathrm{x}, \xi)=\tilde{\mathrm{a}}(\mathrm{x}, \delta(\mathrm{x}) \xi)$. Define $\mathrm{a} \psi \mathrm{DO} \quad \mathrm{K}=\mathrm{K}_{\zeta}(\mathrm{x}, \mathrm{z}), \mathrm{z}=\mathrm{x}-\mathrm{y}$, with symbol $\frac{1}{\zeta-a(x, \xi)}$, a parametrix of $\left(\zeta-A_{0}\right)$,

$$
K(x, z)=(2 \pi)^{-n / 2} \int \frac{e^{i \xi \cdot z}}{\zeta-a(x, \xi)} d \xi
$$

LEMMA 1. Let $a(x, \xi)=\tilde{a}(x, \delta(x) \xi)$ and $b(x) \in L_{w^{\prime}}^{P_{\alpha}}$ satisfy assumptions (2.1) - (2.5) of $\S 2$, then for all $\zeta \in$ range $a(x, \xi)=\mathbf{R}_{+}$, the operator $\mathrm{BK}_{\zeta}$ is $\mathrm{L}^{\mathrm{p}}$-bounded, $1<\mathrm{p} \leqslant \mathrm{p}_{\alpha}$, and its norm is estimated as follows

$$
\left\|\mathrm{BK}_{\zeta^{\prime}}\right\| \leqslant \frac{\mathrm{C}}{|\sin \theta / 2|^{\mathrm{n}+1}} \mathrm{r}^{-1+\mathrm{d} / \mathrm{m}} ; \zeta=\mathrm{re}^{\mathrm{i} \theta}, \mathrm{d}=\mathrm{d}_{\alpha}=\frac{\mathrm{n}}{\mathrm{p}_{\alpha}}+|\alpha| \leqslant \mathrm{m}
$$

where $C$ depends on $p$; the $L_{w}{ }^{p}{ }^{\alpha}$-norm of $b(x)$ and the leading symbol $\mathrm{a}(\mathrm{x}, \xi)$.

PROOF. The operator $B K$ consists of a $\psi D O D^{\alpha} K$ followed by a multiplication with $b(x)$. We start with $D^{\alpha} K$. By the usual product formula of WO's its symbol is equal

$$
\tau(x, \xi)=\sum\left(\frac{\alpha}{3}\right) \xi^{\alpha-\beta} \partial_{x}^{\beta}\left(\frac{1}{\zeta-a}\right) .
$$

We apply the "iterated chain rule" to partial derivatives of $\left(\frac{1}{\zeta-a}\right)$

$$
\partial_{x}^{\beta}\left(\frac{1}{\zeta-a}\right)=\sum C_{B^{1}} \ldots \beta^{k}(\zeta-a)^{-k-1} \prod_{1}^{k}\left(\partial_{x}{ }^{{ }^{j}} a\right),
$$


summation taken over all partitions $B=\sum_{1}^{k} B^{j}$ into the sum of multi-indices $\left\{\varepsilon^{j}\right\}$ with certain combinatorial coefficients ${ }^{C}{ }_{B}{ }^{1} \ldots \beta^{k}$
represented as

$$
\sum \phi_{i}(x) \sigma_{i}(x, \xi)
$$

where

$$
\varphi_{i}(x)=\prod_{1}^{k}\left(\beta^{j}\right) \quad-\begin{aligned}
& \text { product of } k \text { partial derivatives of the } \\
& \text { coefficients of } a(x, \xi)
\end{aligned}
$$

and

$$
\sigma_{i}(x, \xi)=\frac{\xi^{\nu}}{(\zeta-a)^{k+1}}, \quad \begin{aligned}
& \text { where the multiindex } \\
& |v|=k m+|\alpha-\beta|
\end{aligned} \quad \begin{aligned}
& \nu \sum_{1}^{k} \alpha^{j}+(\alpha-\beta)
\end{aligned}
$$

In order to treat a possible $L^{p}$-type singularity of the coefficient $t(x)$ we shall use two interpolation inequalities for convolution and multiplication.

$$
\begin{aligned}
& \|\mathrm{K} * \mathrm{f}\|_{\mathrm{p}^{\prime}} \leqslant\|\mathrm{K}\|_{\mathrm{q}}\|\mathrm{f}\|_{\mathrm{p}} ; \frac{1}{\mathrm{p}^{\prime}}=\frac{1}{\mathrm{p}}-\left(1-\frac{1}{\mathrm{q}}\right) \quad \text { (Young) } \\
& \|\mathrm{bf}\|_{\mathrm{p}^{\prime}} \leqslant\|\mathrm{b}\|_{\mathrm{r}}\|\mathrm{f}\|_{\mathrm{p}^{\prime}} ; \frac{1}{\mathrm{p}^{\prime}}=\frac{1}{\mathrm{p}}+\frac{1}{\mathrm{r}} \quad \text { (Hölder) }
\end{aligned}
$$

including a stronger version of Young's inequality the Hardy-Littlewood-Sobolev inequality, where $K \in L^{q}$-weak (see for instance [17], Ch. 3). The relation between $p, p^{\prime}$ and $q$ is the same but $p=1$ is excluded in the latter case. Rephrasing (3.7) in the reciprocal scale $\frac{1}{p} \in[0,1]$, one can say that a convolution with $K \in L^{q}$ (or $L^{q}$-weak) shifts the $L^{p}$-class to the left by the amount $\left(1-\frac{1}{q}\right)$, while a multiplication with $b \in L^{r}$ shifts it to the right by $\frac{1}{r}$. The product of two operations $f \rightarrow b K * f$ is bounded if the $L^{p}$-classes of $b$ and $K$ are "Young-dual": $\frac{1}{r}=1-\frac{1}{q}$.

This relation explains the limitation on the scale of $L^{p}$-spaces in Lemma 1 , $p \leqslant p_{\alpha}$ (we do not want a "convolution-type" term $K$ to "push" the $L^{p}$-class of $f$ out of the scale $[0,1])$. It also explains the definition of the "fractional order" (2.3). Indeed, a multiplication with $b \in L^{p}$ is "equivalent" by (3.7) to a convolution with the "fractional Laplacian" $\Lambda^{s}=(-\Delta)^{s / 2} ; s=\frac{n}{p}$ ).

Now we return to the operator $b(x) D^{\alpha} K$ and want to make the above heuristic argument accurate. It is convenient to multiply and divide $B K$ by the fractional Laplacian $\Lambda^{s}, s=\frac{n}{p_{\alpha}}\left(s=0\right.$, of $\left.p_{\alpha}=\infty\right)$. Then we get from (3.5)

$$
B K l^{s} \Lambda^{-s}=\sum\left(b \phi_{i}\right) M_{i} \Lambda^{-s}
$$

a combination of multiplication operators $\left(b \phi_{i}\right), \psi D O$ 's $M_{i}$ with symbols

$$
\sigma_{i}=\frac{\xi^{v} \mid \xi^{\prime s}}{(\zeta-a)^{k+1}}
$$

and a fractional Laplacian $\Lambda^{-s}$. The order of $\sigma_{i}$ is $|v|+s-m(k+1)$, which 
by $(3.6)$ is equal to

$$
\ell=|\alpha|-|\beta|+\frac{n}{p_{\alpha}}-m=d_{\alpha}-m-|\beta| \leqslant 0
$$

We "pull out" the absolute value of complex $\zeta=\mathrm{re}^{i \theta}$ in the denominator of (3.8), and by the homogenuity of a write $\sigma_{i}$ in terms of a "uniformly elliptic" symbol $\tilde{\sigma}_{i}=\frac{\xi^{\nu}|\xi|^{\zeta}}{e^{i \theta}-\tilde{a}(x, \xi)}$,

$$
\sigma_{i}(x, \xi)=\frac{r^{\mu}}{\delta^{t}} \tilde{\sigma}_{i}\left(x ; r^{-1 / m} \delta(x) \xi\right)
$$

where

$$
\begin{aligned}
& \mu=-1+\mathrm{d} / \mathrm{m}-|\beta| / \mathrm{m} ; \quad \mathrm{d}=\mathrm{d}_{\alpha}=\frac{\mathrm{n}}{\mathrm{p}_{\alpha}}+|\alpha| \\
& \mathrm{t}=|\nu|+\mathrm{s}=\mathrm{mk}+\mathrm{d}-|\beta| .
\end{aligned}
$$

The weight $\delta^{-t}(x)$ will joint the left (multiplication) factor of $\left(b \phi_{i}\right) M_{i} \Lambda^{-s}$. Remembering that $\phi_{i}$ is a product of derivatives $\prod_{\alpha^{j}}\left(\beta^{j}\right), \sum_{1}^{k} \beta^{j}=\beta$ we get

$$
\begin{aligned}
& b \cdot \prod_{1}^{k} a_{\alpha^{j}}^{\left(\beta^{j}\right)} \\
& \frac{1 \alpha^{j}}{\delta^{d+k m-|\beta|}}=\left(b / \delta^{d}\right) \prod\left(a_{\alpha}{ }^{\left(\beta^{j}\right)} / \delta^{m-\left|\beta^{j}\right|}\right) \text {. }
\end{aligned}
$$

By hypothese $(2.1),(2.2),(2.5)$ of $\S 2$ the first factor $b / \delta^{d}$ is $L^{p} a$, whereas all terms of the product are $L^{\infty}$. Thus the "multipiication: (left) factor $\psi_{i}=\frac{\left(b \phi_{i}\right)}{\delta^{t}} \in L^{p_{\alpha}}$ and the "convolution" (right) factor $\Lambda^{-s}$ of the product $\psi_{i} M_{i} \Lambda^{-s}$ "complete" each other in the sense of interpolation formulas (3.7). It remains to study the middle term. We shall show that a $\psi D O M_{i}$ is $\mathrm{L}^{\mathrm{P}}$-bounded $(1<\mathrm{p}<\infty)$ and estimate its norm.

We first observe that the dilation of symbols $\tilde{\sigma}_{i} \rightarrow \sigma_{i}=\tilde{\sigma}(x, \rho \xi)$ with $\rho=r^{-1 / m_{\delta}}$, results in the dual $L^{1}$-dilation of kernels, $i . e$.

$$
M_{i}(x, z)=\rho^{-n} \tilde{M}_{i}\left(x ; \rho^{-1} z\right)
$$

which represents $M_{i}$ in terms of the "uniformly elliptic" kernel $\tilde{M}_{i}$.

The order $\ell$ of a $\psi D O \sigma_{i}$ is nonpositive by (3.9). So two cases are possible.

$1^{\circ}$. Strictly negative order, $2<0$.

A negative order $\psi D O \quad \tilde{\sigma} \quad S_{1,0}^{l}$ is well known to have an $L^{1}$-radial convolution-type bound

$$
\left|\tilde{M}_{i}(x, z)\right| \leqslant c H(|z|)
$$


where $c$ depends on a finite number $(N=n+1)$ symbol class seminorms of $\tilde{J}$ (see for inst. [5]).

A straightforward evaluation of seminorms yields

$$
\sup _{x, \xi}|\xi|^{N}\left|\left(\partial_{\xi}^{\beta} \tilde{\sigma}_{i}\right)(x, \xi)\right| \leqslant \frac{C}{|\sin \theta / 2|} N, N=|\beta|,
$$

whence the constant in estimate (3.2) of Lemma 1.

From (3.11), (3.12) it follows that the kernel $M_{i}(x, z)$ is bounded by the so-called $p$-dilation of the radial $L^{l}$-function $H$ with $p=p(x)=r^{-1 / m} \delta(x), \quad i . e$.

$$
\left|M_{i}(x, z)\right| \leqslant c_{p}-n(x) H\left(\left|p^{-1}(x) z\right|\right)=H_{p} ; z=x-y .
$$

If $\rho$ were bounded $o(x) \leqslant \varepsilon$ (uniformly elliptic case), then $H_{\rho} \leqslant H_{c}$ and the usual $L^{1}$-convolution would immediately imply the result: $L^{p}$-boundedness of $M_{i}$ for all $1 \leqslant p \leqslant \infty$ (cf. [4], [5]). In case of unbounded $\delta(x)$ the maximal function estimate applies,

$$
\left(H_{\rho} f\right)(x) \leqslant\|H\|_{1} E^{*}(x) .
$$

This yields $\mathrm{L}^{\mathrm{P}}$-boundedness of $\mathrm{M}_{i}$ in all spaces $1<\mathrm{p}<\infty$ and proves Lemma 1 in the first case.

$2^{0}$. Order $\ell=0$. We split $\tilde{\sigma}_{i} \in S_{1,0}^{0}$ into the sum of 3 terms: $\tilde{\sigma}_{i}=c(x) I+\sigma_{0}+\sigma_{-}$, a multiple of the "identity" (in the $\xi$-variable) function; a homogeneous of order zero (in $\xi$ ) symbol $\sigma_{0}(x, \xi)$ with the zero mean-value over the unit sphere $|\xi|=1$; and a negative order symbol $\sigma_{-} \epsilon s_{1,0}^{-\ell}$.

Let us illustrate this splitting for $k=0$, i.e. $\tilde{u}=\frac{\xi^{\nu}}{\zeta-\tilde{a}}(|\nu|=m)$. Then

$$
\tilde{\sigma}=\frac{\xi^{\nu}}{\tilde{a}}-\frac{\zeta_{j} \xi^{\nu}}{(\zeta-\tilde{a}) \tilde{a}}
$$

the first term splits into the sum of $c(x)=\int_{|\xi|=1} \xi^{\nu} / \tilde{a}(x, \xi) d \xi$ and $\sigma_{0}=\xi^{\nu} / \tilde{a}-c(x)$, while the second is obviously in $s_{1,0}^{-m}$. Notice that $\tilde{j}_{-}$is sufficiently regular at $\{0\}$, which is important for the existence of "global" radial bound $H$ of the kernel $\tilde{M}_{-}=\tilde{\sigma}_{-}(x, D)$ (cf. [5]).

Correspondingly kernel $\tilde{M}$ splits into the sum of three kernels: $c(x) I+\tilde{M}_{0}+\tilde{M}_{-}$, a multiplication with "nice" (bounded) function $c(x)$; a Calderon-Zygmund kerne1 $\tilde{M}_{0}(x ; z)$ (homogeneous of degree $-n$ in $z$ ) and an $L^{1}-$ radially bounded kernel $\tilde{M}_{-}$.

The first two of them are invariant under the $p$-dilation

$$
M(z) \rightarrow \rho^{-n} M\left(\rho^{-1} z\right),
$$

while the third is dominated by the maximal function as in case $1^{\circ}$. This completes the proof. 
4. RESOLVENT KERNELS OF $\mathrm{A}_{0}$ AND A AND THEIR APPLICATIONS.

After Lemma 1 we can study the convergence or series (1.5) and (1.6), that give resolvent kernels $R^{0}=\left(\zeta-A_{0}\right)^{-1}$ and $R=(\zeta-A)^{-1}$. We start with $R^{0}$.

THEOREM 1. Series (1.5) converges absclutely in the complement of a parabolic region $\Omega$ about positive real axis in $\mathbb{C}$, in all $L^{\mathrm{P}}$-spaces $1<\mathrm{p}<\infty$, and defines the resolvent $\mathrm{R}_{\zeta}^{0}$ of $\mathrm{A}$.

PROOF. We recall that the operator $L=\left(\zeta-A_{0}\right) K-I$. By the product fcrmula of $\psi$ 'DO's its symbol

$$
\sigma_{L}(x, \xi)=\sum_{1 \leqslant|\beta| \leqslant m} \frac{1}{\beta !} D_{\xi}^{\beta}(a) \partial_{x}^{\beta}\left(\frac{1}{\zeta-a}\right),
$$

expands into the sum $\sum \phi_{i} \sigma_{i}$ of the type that appear in Lemma 1. Namely,

$$
\phi_{i}(x)=a_{\alpha} \prod_{1}^{k} a_{\alpha^{j}}^{\left(\beta^{j}\right)} ; \sum_{1}^{k} \beta^{j}=\beta ; \quad\left(a_{\alpha} \in L_{\delta^{-m}}^{\infty} \text { plays the role of } b(x)\right)
$$

and

$$
\sigma_{i}(x, \xi)=\frac{\xi^{\nu}}{(\zeta-a)^{k+1}} ; \nu=\alpha-\beta+\sum_{1}^{k} \alpha^{j} ;|\nu|=(k+1) m-|\beta| \text {. }
$$

So the whole argument of Lemma 1 can be repeated for $\sigma_{L}$. Notice that all $\psi$ DO's $\sigma_{i}(x, D)$ have strictly negative order, $\leqslant-1$.

Hence by Lemma 1 each term $\phi M$ of $L$ and consequently the whole operator L is estimated as

$$
\|L\| \leqslant \sum\left\|\phi_{i} M_{i}\right\| \leqslant \frac{C}{|\sin \theta / 2|^{n+1}} r^{-\lambda} ; \lambda=1-|\beta| / m \geqslant \frac{1}{m},
$$

with $\mathrm{C}$ depending on $\mathrm{L}^{\infty}$-norms of $\phi_{i}$ and $\mathrm{p} \quad(1<\mathrm{p}<\infty)$. Returning to series

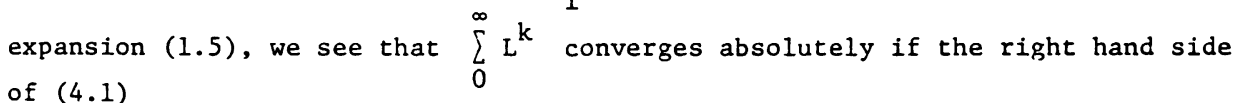

$$
\frac{\mathrm{C}}{|\sin \theta / 2|^{\mathrm{n}+1}} \mathrm{r}^{-\lambda}<1
$$

This condition gives a parabolic region $\Omega$ about positive real axis in $\mathbb{C}$, in whose complement we have a well defined bounded operator $R_{\zeta}^{0}=K_{\zeta}\left(I-L_{\zeta}\right)^{-1}$.

We want to show that $R_{\zeta}^{0}$ is the resolvent of $A_{0}$, that is

(I) $\quad\left(\zeta-A_{0}\right) R_{\zeta}^{0} f=f ; \quad f \in L^{p}$

(II) $\quad \mathrm{R}^{0}\left(\zeta-\mathrm{A}_{0}\right) \mathrm{f}=\mathrm{f}$; for all $\mathrm{f}$ in the $L^{\mathrm{P}}$-domain of $\mathrm{A}_{0}$.

The first formula immediately follows from the relation $\left(\zeta-A_{0}\right) K=I-L$. It shows that $R^{0}$ is the right resolvent of $A_{0}$. To show the existence of the left resolvent (II) we shall use duality between $L^{P}$-spaces. Namely: the adjoint of the right resolvent of $A_{0}$ in $L^{p}$ becomes the left resolvent of the adjoint operator $A_{0}^{*}$ in $L^{P^{\prime}}\left(\frac{1}{p^{\prime}}+\frac{1}{p}=1\right)$, and vice versa.

We can not apply the above result directly to $A_{0}^{*}$ to show the existence of the right resolvent, since $A_{0}^{*}$ is not of homogeneous order. But $A_{0}^{*}=A_{0}+B$, whose lower order terms $\left.\left\{b_{\alpha} D^{\alpha}\right\}\right|_{\alpha} \leqslant m$ have coefficients bounded "relative to $A_{0}$ ". 
Therefore the "perturbation" Theorem (Theorem 4 below) applies to prove the existence of the right resolvent for $A_{0}^{*}$ outside of another parabolic region $?^{\prime}$. Once both resolvents, right and left, are shown to exist they must be equal. This completes the proof of Theorem 1 .

Next we apply Lemma 1 to characterize the $L^{p}$-domain of $A_{0}, D_{p}\left(A_{0}\right), 1<p<\infty$. For constant coefficient elliptic operators $D_{p}\left(A_{0}\right)$ is known to be the $m^{-t h}$ Sobolev space $L^{\mathrm{p}, \mathrm{m}}=(1-\Delta)^{\mathrm{m} / 2} \mathrm{~L}^{\mathrm{p}}$ and the same is true for uniformly elliptic operators (see [4]). For strictly elliptic operators of the above type we have

THEOREM 2. The $\mathrm{L}^{\mathrm{P}}$-domain of $\mathrm{A}_{0}$ is equal to the $\mathrm{m}^{- \text {th }}$ weighted Sobolev space with the weight $w=\delta^{m}$

$$
L_{\mathrm{w}}^{\mathrm{p}, \mathrm{m}}=\left\{\mathrm{f}: \sum_{|\alpha| \leqslant \mathrm{m}} \int\left|\mathrm{wf}{ }^{(\alpha)}\right|^{\mathrm{p}} \mathrm{dx}<\infty\right\},
$$

and the norms $\left\|\left(\zeta-A_{0}\right) f\right\|$ in $D_{p}\left(A_{0}\right)$ and $\sum_{|\alpha| \leqslant m}\left\|w D^{\alpha} f\right\|_{p}$ in $L_{w}^{p, m}$ are equivalent.

PROOF. We have to show that the operator $w(1-\Delta)^{m / 2} R_{\zeta}^{0}$ is bounded and invertible in $L^{p}$. But $R_{\zeta}^{0}=K(I-L)^{-1}$. It suffices to show boundedness and invertibility of the operator $T=w(1-\Delta)^{\mathrm{m} / 2} \mathrm{~K}$. Boundedness follows directly from Lemma 1. Indeed

$$
w(1-\Delta)^{\mathrm{m} / 2} \mathrm{~K}=\sum_{|\alpha| \leqslant \mathrm{m}} c_{\alpha} \mathrm{wD}^{\alpha} \mathrm{K}
$$

is the sum of terms that appear in Lemma 1.

To prove invertibility it is convenient to use $(\lambda-\Delta)^{\mathrm{m} / 2}$ for sufficiently large $\lambda>0$ instead of $(1-\Delta)^{m / 2}$ in the definition of $L_{w}^{p, m}$ - norm and also to reverse two terms in the product $w(\lambda-\Delta)^{\mathrm{m} / 2}$.

We observe that

$$
w(\lambda-\Delta)^{m / 2}=\left[(\lambda-\Delta)^{m / 2}+B\right] w,
$$

where order $B \leqslant m-1$, and the coefficients $b_{\alpha}=\sum_{|\nu| \leqslant m} c_{v} w^{(\nu)} / w$ are ail

bounded. Then for sufficiently large $\lambda$ the operator norm

$$
\left\|B(\lambda-\Delta)^{-m / 2}\right\|<1 / 2 \text {. }
$$

Hence the inverse $\left[(\lambda-\Delta)^{\mathrm{m} / 2}+B\right]^{-1}$ exists and is bounded.

Now we can invert $T=w(\lambda-\Delta)^{m / 2} R^{0}$ using (4.3),

$$
\mathrm{T}^{-1}=\left(\zeta-\mathrm{A}_{0}\right) \mathrm{w}^{-1}\left[(\lambda-\Delta)^{\mathrm{m} / 2}+\mathrm{B}\right]^{-1} \text {. }
$$

But $A_{0} w^{-1}=\tilde{A}_{0}+\tilde{B}$ with uniformly elliptic $\tilde{A}_{0}=w^{-1} A_{0}$ and $\tilde{B}$ of the same type as $B$.

Finally "pulling out" $(1-\Delta)^{\mathrm{m} / 2}$ from the right factor in $(4.4)$, $(\lambda-\Delta)^{-m / 2}\left[I+(\lambda-\Delta)^{-m / 2} B\right]^{-1}$ the problem reduces to $L^{p}$-boundedness of the 
operator $S=\left(\tilde{A}_{0}+\tilde{B}\right)(\lambda-\Lambda)^{-m / 2}$ for uniformly elliptic $\tilde{A}_{0}$ and "relatively small" $\tilde{B}$, which is well known (cf. [4]). Indeed, the operator $S$ is a combination of zero- and negative order $\psi$ DO's $\left\{\tilde{a}_{\alpha}(x) D^{\alpha}(\lambda-\Delta)^{m / 2}:|\alpha| \leqslant m\right\}$. The former are given by Calderon-Zygmund kernels, hence ${ }_{L^{p}}$ for all $1<p<\infty$ (see [17], ch. 2). The latter have $L^{1}$-radially bounded kernels, hence $L^{p}$ for all $1 \leqslant p \leqslant \infty$.

In many applications, including the perturbation series (1.6), one needs to estimate the operator norm of $\mathrm{BR}_{\zeta}^{0}$. By Theorem $1 \quad \mathrm{R}_{\zeta}^{0}=\mathrm{K}_{\zeta}\left(\mathrm{I}-\mathrm{L}_{\zeta}\right)^{-1}$ and $\left(I-L_{\zeta}\right)^{-1}$ is easily shown to converge to $I$ as $\zeta \rightarrow \infty$ uniformly in any sector $\{|\arg \zeta| \geqslant \theta>0\}$. The problem is thus reduced to estimating $\left\|\mathrm{BK}_{\zeta}\right\|$. The latter is provided by Lemma 1 .

COROLLARY 1. If $A_{0}$ and $B$ satisfy assumptions of $\S 2$, then

$$
\left\|B R_{\zeta}^{0}\right\| \leqslant\left(C \sum\left\|b_{\alpha}\right\|\right) \frac{r^{-1+d / m}}{|\sin \theta / 2|^{k+1}} ;
$$

for all $L^{p}, \quad 1<p \leqslant \min \left\{p_{\alpha}\right\}$ and $\zeta \in \mathbb{M} \Omega$ (where $R_{\zeta}^{0}$ exists). Here $d=\max \left\{d_{\alpha}\right\}$ is the "fractional order of $B$, constant $C$ depends on $P$ and the symbol $a(x, \xi)$.

From (4.5) immediately follows

COROLLARY 2 (cf. [5], [4], [16]). The operator $B$ is $A_{0}$-bounded and the $L^{P}$-domains of $A$ and $A_{0}$ are equal

$$
D_{p}(A)=D_{p}\left(A_{0}\right)=L_{w}^{p, m} ; w=\delta^{-m}, 1<p \leqslant \min \left\{p_{\alpha}\right\} .
$$

Let us notice that the relative bound in the right hand side of $(4.5)$ can be made as small as one likes, taking sufficiently large $r$, provided the "fractional" order $d<m$. In this case the perturbation series (1.6) converges absolutely in the complement of a parabolic region

$$
\Omega^{\prime}=\{\zeta \text { : relative bond } \leqslant 1\} \text {. }
$$

The limit case, $d=m$, is important, as it gives the "optimal amount" of $L^{p}$-type singularity of lower order coefficients and also allows "top order" perturbations. In this case one can claim somewhat less.

PROPOSITION 1. Operator $\left\|B_{\zeta}^{0}\right\|<1$ in any region

$$
\Omega_{R, \theta}=\{\zeta:|\zeta| \geqslant R ;|\arg \zeta|>\theta\}
$$

with arbitrarily small $\theta$ and large $R$, depending on $\theta$.

To prove Proposition 1 we cut each coefficient $b_{\alpha} \in L^{p_{\alpha}}$ into two parts: $b_{\alpha}^{\prime} \in L^{\infty}$ and "very small" $b_{\alpha}^{\prime \prime} \in L^{p}, \| b_{\alpha}^{\prime \prime \prime}<\varepsilon$. Consequently, the whole perturbation $B$ splits into the sum $B^{\prime}+B^{\prime \prime}$. The first term has order $d \leqslant m-1$, hence $\left\|B^{\prime} R_{\zeta}^{0}\right\|$ becomes small outside of a parabolic region $\Omega^{\prime}$, while the second $\left\|B^{n} R_{\zeta}^{0}\right\|<1$ in some region $\Omega_{R, 9}$. Here $\theta$ depends on $\left[\left\|b_{\alpha}\right\|\right.$ and can be made as small as one likes. This proves the Proposition.

From Proposition 1 easily follows 
COROLLARY 3 (a priory estimate). For any $\varepsilon>0$ there exists $C=C_{\varepsilon}>0$ s.t.

$$
\|B f\|_{p} \leqslant \varepsilon\left\|_{0} f\right\|_{p}+C\|f\|_{p} ; \quad f \in L_{w}^{p, m}
$$

Along with Kato-Rellich Theorem a priory estimate (4.7) implies essential selfadjointness in $L^{2}$ of a formally symmetric operator $A_{0}+B$. Proposition 1 also yields a large class ("optimal" in the sense of $L^{p}$-type singularity of coefficients) of "well defined" elliptic operators in $\mathrm{L}^{\mathrm{P}}$-spaces. Namely,

THEOREM 3. 1) If an operator $A=\sum_{a} a_{\alpha}(x) D^{\alpha}$ of $\S 1$ has leading coefficients s.t. $\left\{\frac{a_{\alpha}(x)}{\delta^{m}(x)}\right\}_{|\alpha|=m}$ - uniformly continuous, and the lower order coefficients $a_{\alpha} \in L_{w}^{P_{\alpha}}$, satisfy (2.3) - (2.4), then $A$ is "well defined" in all $L^{\mathrm{p}}$-spaces $1<\mathrm{p} \leqslant \min \mathrm{p}_{\alpha}$ in the sense that

$$
D_{p}(A)=L_{w}^{p, m} \text { and } A \mid L_{w}^{p, m}=\text { closure }\left\{A_{m i n}=A \mid C_{0}^{\infty}\right\}
$$

2) If $A$ is formally symmetric and $\min \left\{p_{\alpha}\right\} \geqslant 2$ then $A$ is essentially selfadjoint on $C_{0}^{\infty}$.

Theorem 3 extends many of earlier known results [8], [9], [24], [4], [11], [15], [16] on essential selfadjointness and $L^{P}$-theory.

Now we turn to the resolvent $R_{\zeta}$ of $A$.

THEOREM 4. (cf. [5], [4]) Let $A=A_{0}+B$ be an operator of Theorem 3 . Then

(I) for all $1<\mathrm{p} \leqslant \min \left\{\mathrm{p}_{\alpha}\right\}$ the resolvent $\mathrm{R}_{\zeta}=(\zeta-\mathrm{A})^{-1}$ exists in $\Omega_{\mathrm{R},}$ ? and is given by an absolutely convergent series (1.6).

(II) (resolvent summability) for all $f \in L^{p}\left(1<p \leqslant \min \left\{p_{\alpha}\right\}\right)$ the family

$$
\left(\zeta R_{\zeta} f\right)(x) \rightarrow f(x) \text { as } \zeta \rightarrow \infty \text { uniformly in } \Omega^{\prime}
$$

in $L^{\mathrm{P}}$-norm.

The first statement is already proved, the second follows from the estimates of Lemma 1 (cf. [4], [5]).

After Theorem 2 we can obtain a variety of other "analytic multipliers" $\phi(A)$ and "summation families" $\left\{\phi_{\varepsilon}(A)\right\}_{\varepsilon}$ by Cauchy integration of $R_{\zeta}$. Indeed, the formula

$$
\phi(A)=\frac{1}{2 \pi i} \int_{\Gamma} \phi(\zeta)(\zeta-A)^{-1} \mathrm{~d} \zeta
$$

defines a nice (boundel) operator $\phi(A)$, provided $\phi$ is integrable over the contour I with respect to the measure $d \mu=\frac{\mathrm{Cr}^{-1+d / m}}{|\sin \theta / 2|^{n+1}} d \zeta$. One such example is the family of functions $\left\{\phi_{t}(\zeta)=e^{-t \zeta}\right\}_{t}$. Here contour $\Gamma$ consists of two rays $\left\{r e^{ \pm i \psi} ; r>r_{0}\right\}, 0<\psi \leqslant \frac{\pi}{2}$ and a circle $\left\{r_{0} e^{i e}:|\theta| \geqslant \psi\right\}$. Angle $\psi$ can be chosen arbitrarily small due to the shape of $\Omega_{R, \theta}$.

Thus we get 
THEOREM 5. An operator A of Theorem 3 generates a holomorphic semigroup $e^{-t A}$ in the right half plane $\operatorname{Re} t>0$ in all $L^{p}, 1<p \leqslant \min \left\{p_{\alpha}\right\}$.

Moreover, the family of functions $e^{-t A} f(x) \rightarrow f(x)$ as $t \rightarrow 0$ in $L^{p}$-norm.

REMARK 1. As in [4] ( $(5)$, all the above results extend to strictly elliptic operators on certain Riemannian manifolds diffeomorphic to $\mathbb{R}^{\mathbf{n}}$ and strictly elliptic systems.

REMARK 2. More general classes of coefficients $\left\{b_{\alpha}\right\}$ of the perturbation $B$ can be allowed in $\S 3$. One such class consists of $b_{\alpha}(x)$ defined on quotient spaces $E=\mathbb{R}^{n} / E \quad\left(E\right.$ is a subspace of $\mathbb{R}^{n}$ ), which are $L^{p}$ on $E$, like "N-body" potentials: $\quad V=\sum_{i<j} V\left(x^{i}-x^{j}\right)$ on $R^{3 N}\left(x^{i} \in \mathbb{R}^{3}\right)$. In this case condition (2.4) of $\S 2$ should read: $\frac{\operatorname{dim} E}{\mathrm{P}_{\alpha}}+|\alpha| \leqslant \mathrm{m}, \quad$ (see [5]). Another extension of $\mathrm{L}^{\mathrm{p}}$ classes is discussed in [15], [16]. It consists of all functions $b_{\alpha}(x)$, whose convolution with $\mathrm{H}_{\alpha}(\mathrm{z})=|z|^{-\mathrm{s}}(|z| \leqslant 1$, and $\mathrm{s}=\mathrm{n}-\mathrm{m}+\alpha)$ (a "local singularity" of $\left.D^{\alpha}\left(\zeta-A_{0}\right)^{-1}\right)$ is bounded. This condition is close to so called "Rollnik condition" in the theory of Schrödinger operators (see [25]).

We shall conclude this section with two examples, which have bearing on $\mathrm{L}^{1}$-theory of the above class of operators. This theory poses the following interesting harmonic analysis problem: given an $L^{1}$ function $K(x)$ and a dilating factor $\delta(x)>0$, when the kernel $K_{\delta}=\frac{1}{\delta^{n}(x)} K\left(\frac{x-y}{\delta(x)}\right)$ is $L^{1}$ bounded.

Two examples below indicate that the situation in $L^{1}$-case becomes more subtle compared to $L^{p}$-theory $(p>1)$. Namely, an $L^{1}$-radially bounded $K$ and $a$ "finitely propagating" $\delta$ are no longer sufficient for $\mathrm{L}^{1}$ : the growth of $\delta$ must be "slower" than $O(|x|)$, and some additional relation between $K$ and $\delta$ appears.

EXAMPLE 1. Take $\delta(x)=|x|$ on $\mathbb{R}$. Then $\mathrm{K}(\mathrm{x}, \mathrm{y})=\frac{1}{\mathrm{x}} \mathrm{K}\left(1-\frac{\mathrm{y}}{\mathrm{x}}\right)$ is homogeneous of degree -1 . It is well known (see, for instance, [17], Appendix) that $K$ is $L^{p}$ if and only if $\int y^{-p_{K}}(1-y)$ dy $<\infty$. The latter is obviously true for $p>1$, but fails for $p=1$.

EXAMPLE 2. Now we take $\delta(x)=2 \sqrt{x}$ on $\mathbb{R}$ and write the operator $k_{\delta} f$ as

$$
\left(K_{\delta} f\right)(x)=\int K(y) f(x-\delta(x) y) d y \text {. }
$$

Then

$$
\|K f\|_{1}=\int K(y) \int f(x-\delta(x) y) d x d y .
$$

We introduce a new variable $u=x-\hat{o}(x) y=p_{y}(x)$. The range of $u$ is the whole real line $\mathbb{R}$, but the function $u=\phi_{y}(x)$ has a critical point $x_{0}=y^{2}$, and on the interval $-y^{2} \leqslant u \leqslant 0 \quad u$ is "three-fold" (see figure). We divide the line $\mathbf{R}_{\mathrm{x}}$ into three intervals $I_{1}=(-\infty ; 0] ; I_{2}=\left(0 ; 2 y^{2}\right] ; I_{3}=\left(2 y^{2} ;+\infty\right)$. on $I_{1}$ and $I_{3}$ the Jacobian $\left(\frac{d u}{d x}\right)$ is bounded from both sides,

$$
0<c_{1} \leqslant\left(\frac{d u}{d x}\right) \leqslant c_{2}<\infty .
$$


Hence

$$
\int_{I_{1}}+\int_{I_{3}} f(x-o(x) y) d x \leqslant \frac{1}{c_{1}}\|f\|_{1}
$$

It is only a neighborhood of the critical point $x_{0}=y^{2}$ that causes $\left(\frac{d u}{d x}\right)$ to "blow up". The Jacobian $\left(\frac{d u}{d x}\right)$ is computed explicitely.

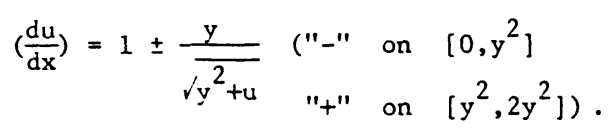

Hence

$$
\int_{I_{2}} f(x-o(x) y) d x=2 \int_{-y}^{0} f(u) \frac{y}{\sqrt{y}+u} d u+2 \int_{-y}^{0} f(u) d u .
$$

Finally, the change of variable $y \rightarrow \sqrt{y}$, and the order of integration yields

$$
\int_{0}^{\infty} K(y) \int_{-y}^{0} f(u) \frac{y}{\sqrt{y^{2}+u}} d u=\int_{0}^{\infty} f(u) \int_{-u}^{\infty} \frac{k(\sqrt{y})}{\sqrt{y+u}} d y d u .
$$

This shows that $K$ is $L^{1}$-bounded iff the convolution $K(\sqrt{y}) * \frac{1}{\sqrt{y}}$ is bounded. Then

$$
\left\|K_{\delta}(f)\right\|_{1} \leqslant\left(\left\|k_{1}+\right\| K(\sqrt{y}) * \frac{1}{\sqrt{y}} \|_{\infty}\right)\|f\|_{1}
$$

A similar condition on $K$ can be derived for other polynomial functions $\delta(x)=|x|^{s}(s<1)$. Namely, the convolution of $y^{1 / 2-s} k\left(y^{1-s}\right)$ and $\frac{1}{\sqrt{y}}$ must

These results can be used to study $L^{1}$ properties of the resolvent kernel $R_{\zeta}$ for operators $A=\delta(x)^{m} \frac{d^{m}}{d x^{m}}+\ldots$ on $\mathbb{R}$. Indeed, the kernel

$K(z)=\int_{-\infty}^{\infty} \frac{e^{i z \cdot \xi}}{\zeta-\xi^{m}} d \xi$ is bounded near $\{0\}$ and decays exponentially at $\{\infty\}^{-\infty}, K(z) \sim \mathrm{e}^{-y|z|}$. If $\delta(x) \sim|x|^{s}$ with $s<m$, the condition $y^{-1 / 2}-s_{K\left(y^{1-s}\right)} * \frac{1}{\sqrt{y}} L^{\infty}$ is obviously satisfied. Hence $K_{\delta}$ and consequently $R_{;}$ are $L^{1}$. On the other hand if $\delta(x) \sim|x|$ has the maximal allowed growth both kernels fail to be $L^{1}$. 


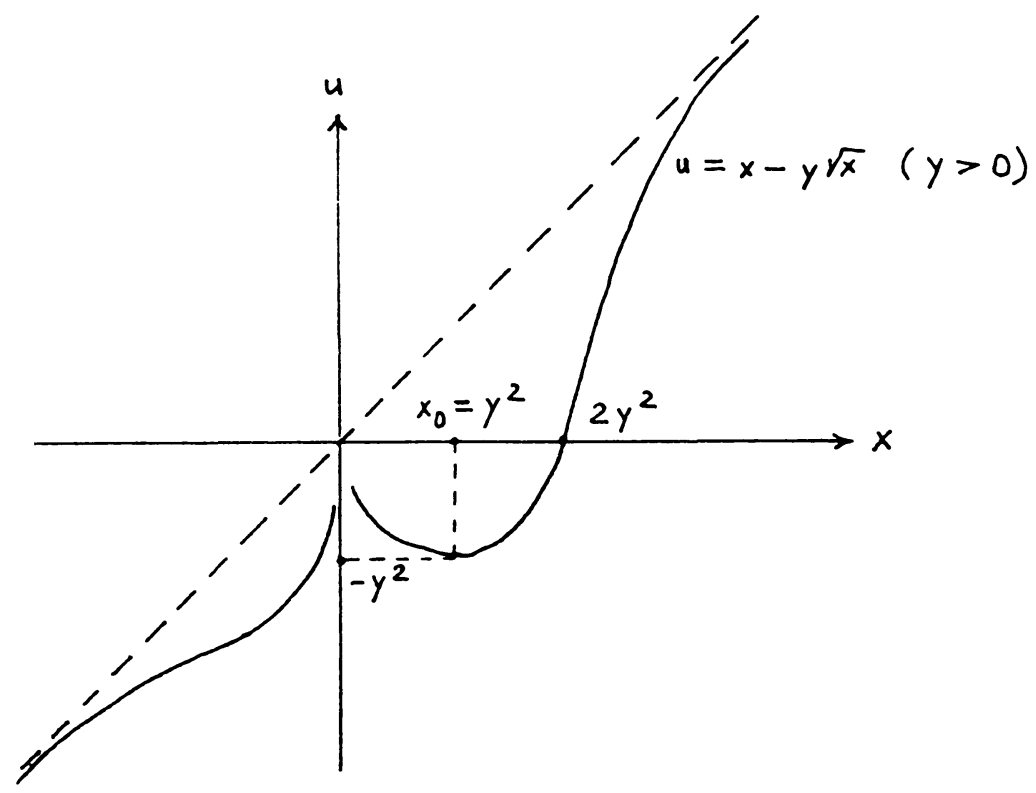

ACKNOWLEDGEMENT. It is my pleasure to thank R. Kaufmann, D. Finch and H. Cordes for stimulating discussions.

\section{REFERENCES}

1. EIDELMAN, S.D. Estimates of solutions of parabolic systems and some of their applications, Math. Sbornik 33 , no. 3 (1953) and $\underline{38}$, no. 1 (1956) (Russian).

2. GELFAND, I.N., SHILOV, G.E. Generalized functions, vol. 3, Moscow, 1958.

3. AGMON, S., DOUGLIS, A., and NIRENBERG, L. Estimates near the boundary of elliptic differential equations satisfying general boundary conditions, Comm. Pure App1. Math. 12 (1959), 623-727.

4. GLRARIE, D. Kernels of elliptic operators: bounds and summability, J. Diff. Equations, 55, no. 1 (1984), 1-29.

5. GURARIE, D., KON, M. Radial bounds for perturbation of elliptic operators, J. Funct. Anal. 56, no. 1 (1984), 99-123.

6. NAGEL, A., STEIN, E. Lectures on pseudodifferential operators: Regularity theorems and applications to non-elliptic problems, Princeton Univ. Press (1979).

7. CODDINGTON, E.A., LEVINSON, N., Theory of ordinary differential equations. McGraw-Hil1, NY, 1955.

8. CHERNOFF, P.R. Essential selfadjointness of powers of generators of hyperbolic equations, J. Funct. Anal. 12, 401-414 (1973).

9. CORDES, H.O. A global parametrix for pseudodifferential operators over $\mathbf{R}^{\mathrm{n}}$ with applications, Preprint.

10. FREHSE, J. Essential selfadjointness of singular elliptic operators, Bol. Soc. Bras. Math. 8.2 (1977), 87-107.

11. KATO, T. Remarks on the selfadjointness problem and related problems for differential operators, Spectral Theory of Differential Operators (Ed. T.W. Knowles, R.I. Lewis) North Holland, 1981.

12. KAUFFMAN, R.M. On the limite-n classification of ordinary differential operators with positive coefficients, Lect. Notes Math. 564, Springer 1980, 259-266. 
13. PARIS, R.B., WOOD, A.D. On the $\mathrm{L}^{2}$-nature of solutions of $n-t h$ order symmetric differential equations and McLeod conjecture, Proc. Roval Soc. Edinburg, 90A (1981) 209-236.

14. CHEEGER, J., GROMOV, M., and TAYLOR, M. Finite propagation speed, kernel estimates for functions of the Laplace operator, and the geometry of complete Riemannian manifold, J. Diff. Geom. 17, 1, (1982), 15-55.

15. SCHECHTER, M. Spectra of partial differential operators, North-Holland, $19: 1$.

16. WEDER, R. The unified approach to spectral analysis, I, Comm. Math. Phys. 60, 291-299 (1980); II, Proc. AMS, 75, 1, (1979), 81-84.

17. SIEIN, E. Singular integrals and differentiability properties of functions, Princeton Univ. Press, 1970.

18. REED, M., SIMON, B. Methods of modern mathematical physics, v. II, Academic Press, 1975.

19. BROWDER, F.E. On the spectral theory of elliptic operators, I, Math. Ann. $142(1961), 22-130$.

20. GARDING, L. Eigenfunction expansions connected with elliptic differential operators, 2nd Skand. Mat. Kongr. Lund, 1953, 44-55.

21. BEALS, R. A general calculus of pseudodifferential operators, Duke Math. Journ. (1975), 1-42.

22. HÖRMANDER, L. The Weyl calculus of pseudodifferential operators, Comm. Pure Appl. Math. 32 (1979), 359-443.

23. BEALS, R. $L^{P}$ and Hölder estimates for pseudodifferential operators: sufficient conditions, Ann. Inst. Fourier, 29, 3, 1979, 236-260.

24. DUNG, N.X. Selfadjointness for general elliptic operators with Sobolev type coefficients, Preprint, 1983.

25. SIMON, B. Quantum mechanics of Hamiltonians, defined as quadratic forms, Princeton Univ. Press, 1971. 


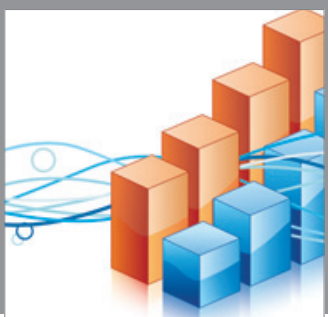

Advances in

Operations Research

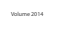

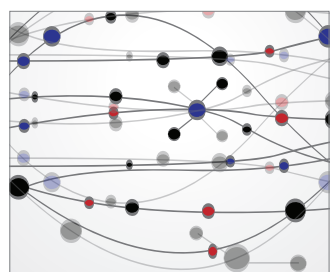

\section{The Scientific} World Journal
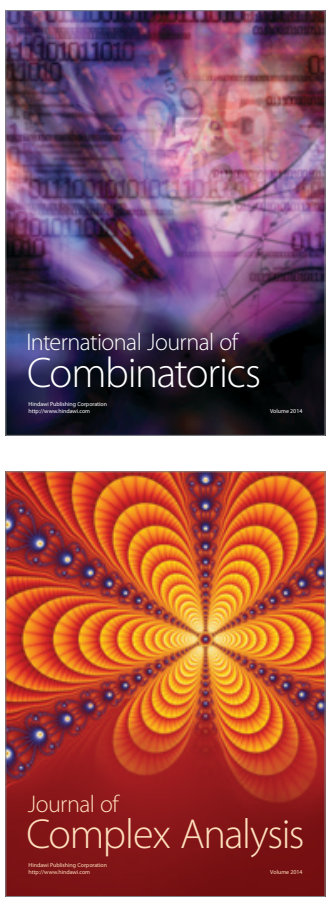

International Journal of

Mathematics and

Mathematical

Sciences
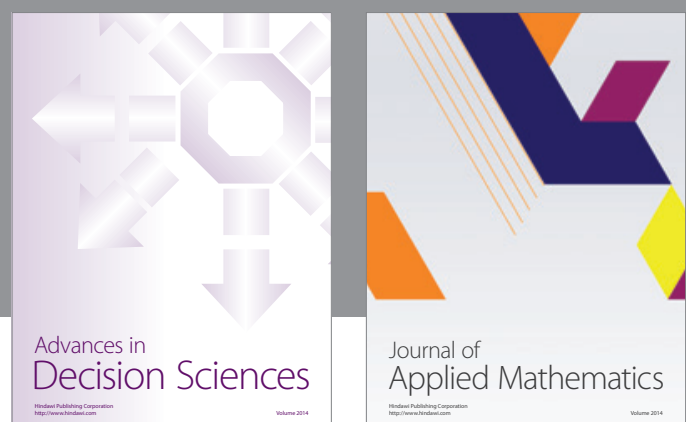

Journal of

Applied Mathematics
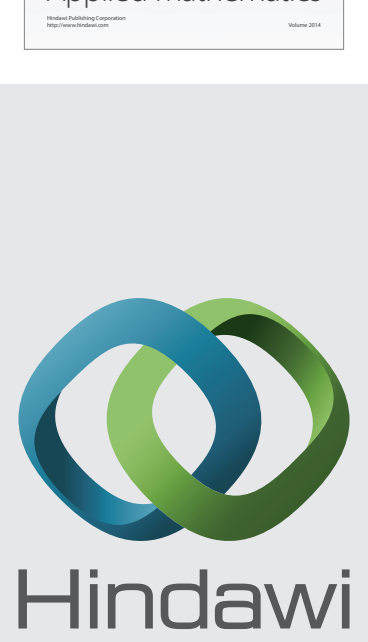

Submit your manuscripts at http://www.hindawi.com
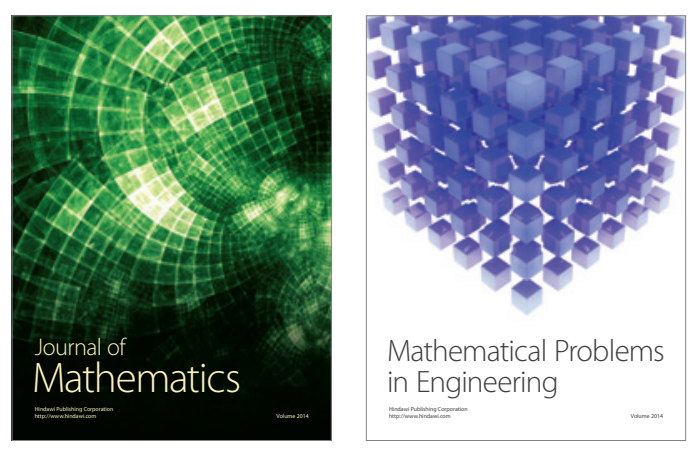

Mathematical Problems in Engineering
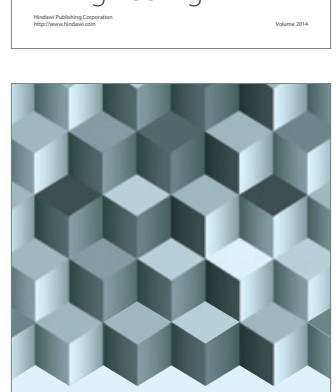

Journal of

Function Spaces
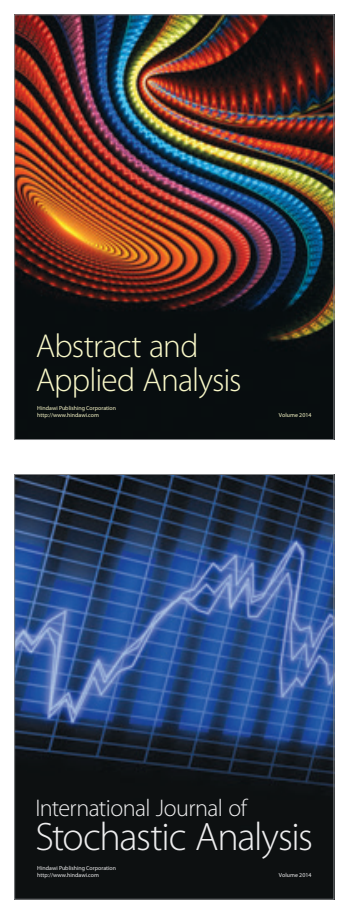

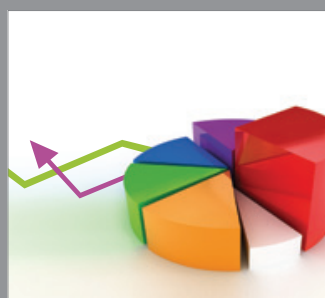

ournal of

Probability and Statistics

Promensencen
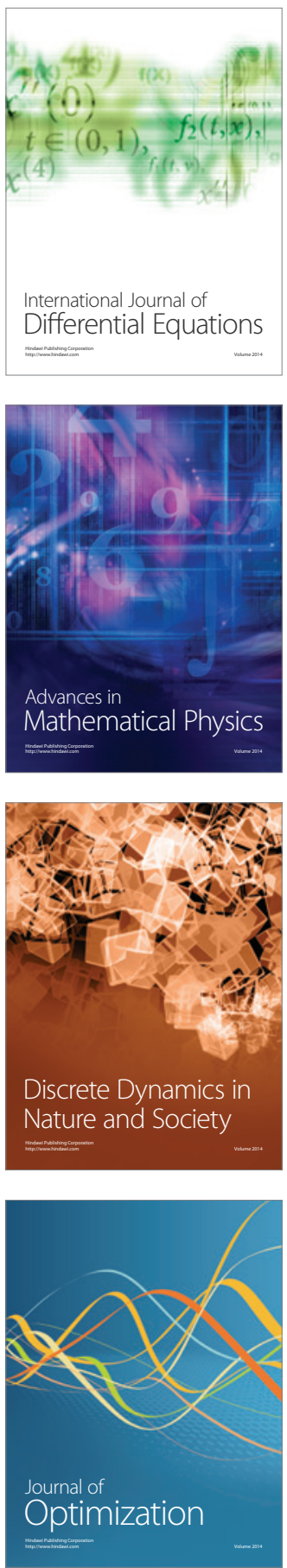